\title{
L'université circonscrite par Bologne
}

\author{
Quand l'Europe impose sa définition de la bonne \\ institution, du bon enseignement et de la bonne \\ science en réseau
}

\section{Sarah Croché}

Keywords : Bologna process, European Commission, university, european triangle, network. Abstract: The Bologna process has (re)launched the debate, consubstantial to European building, on the missions and the governance of universities. It has permitted to the European Commission to take the piloting of higher education and research policies, by putting them to the service of the Lisbon strategy. More its implication in the Bologna process increased, more the Commission has oriented universities' reform. This article proposes a sociobistory of the European universities policies between 1950 and 2009. It traces particularly soft methods by which, in a decade (1998-2009), the Commission reaches them to define in Europe the good university institution, the good teaching and the good science, from now on thinking in network. The aims is to show the shifts in the vision of the universities when the relationship between the actors of the Bologna process were modified.

Mots clés : processus de Bologne, Commission européenne, université, triangle européen, réseaux

Résumé : Le processus de Bologne a (re)lancé le débat, consubstantiel à la construction européenne, sur les missions et la gouvernance des universités. Il a permis à la Commission européenne de prendre le pilotage des politiques d'enseignement supérieur et de recherche, et de les mettre au service de la stratégie de Lisbonne. Plus son implication dans le processus de Bologne a été significative, plus la Commission a pu orienter la réforme des universités. Cet article propose une sociohistoire des politiques universitaires européennes entre 1950 et 2009. Il retrace les méthodes douces par lesquelles, en une décennie (1998-2009), la Commission est parvenue à définir en Europe la bonne institution universitaire, le bon enseignement et la bonne science, désormais pensés en réseau. Il s'agit de mettre en évidence les glissements qui se sont produits dans la vision des universités lorsque les relations entre les acteurs du processus de Bologne se sont modifiées.

Entamée au début des années 1950, la construction européenne n’a jamais été pensée sans provoquer des débats sur l'Université, ses missions et sa gouvernance. Fortement 
engagée à ce moment, la réflexion sur le rôle des universités dans l'avènement d'une Europe supranationale a été quelque peu mise de côté au cours des décennies suivantes, pour revenir sur le devant de la scène à la fin des années 1990 avec le lancement du processus de Bologne en 1998 et de la stratégie de Lisbonne en 2000. Ces événements ont permis à la Commission européenne de prendre le pilotage de la réforme des universités, d'en transformer la vision, la gouvernance et les missions. Cet article propose une sociohistoire qui couvre la période qui s'étend de 1950 à 2009, dans laquelle il s'agit de mettre en évidence les modifications de la vision des universités qui se sont produites en Europe chaque fois que les relations entre la Commission et les autres acteurs du supérieur (organisations internationales, non gouvernementales et États) se sont transformées. L’objectif est d'étudier les méthodes douces qui, entre 1998 et 2009, ont permis à la Commission de mettre les universités au service du projet européen de construire une société de la connaissance compétitive, et d'imposer sa définition de la bonne institution universitaire, du bon enseignement et de la bonne science en réseau. En cinquante ans, le projet de créer une université européenne énoncé en 1957 a laissé place à celui de créer une Europe des universités dans le cadre du processus de Bologne. Les trois missions interdépendantes d' «Éducation, de Recherche et d'Innovation ", qui ont été attribuées en 2005 aux universités en Europe par la Commission européenne, sont celles définies dans le «triangle de la conaissance ».

\section{La politique universitaire européenne de 1950 à 1998}

Au cours des années 1950, l'enseignement supérieur a été considéré comme un des instruments majeurs que les pays pouvaient activer pour s'imposer dans les compétitions qui les opposaient (Croché 2009). Les événements qui ont marqué les politiques du supérieur aux États-Unis dès les années 1950, comme le lancement de Spoutnik (1957) ou la publication du rapport $A$ Nation at Risk (1983), ont contribué à forger et à renforcer l'idée selon laquelle l'enseignement est un outil de compétition internationale. Cette idée a ensuite débordé du continent américain pour toucher les pays européens qui ont eu tendance à s'inspirer des politiques d'enseignement et de recherche nord-américaines depuis la fin de la seconde guerre mondiale. La compétition a alors contribué à faire évoluer les systèmes d'enseignement de manière assez similaire. Meyer et al. (1977) ont ainsi montré qu'une culture mondiale de l'éducation est apparue, due aux caractéristiques du système mondial contemporain, qui affectent tous les États simultanément. L'expansion de l'enseignement supérieur a été envisagée comme la conséquence de stratégies engagées par chaque État, opérant dans une économie capitaliste mondiale dans laquelle le développement est lié à la compétition économique. En Europe, certains États ont ainsi voulu donner à la Commission européenne la possibilité d’intervenir dans le supérieur et d'orienter les politiques 
universitaires nationales pour renforcer les États dans les compétitions dans lesquelles ils étaient inscrits. Même si une grande partie de la littérature a considéré que les Communautés ne se sont pas intéressées au supérieur avant 1970 et que leurs premières initiatives datent de 1971, Corbett (2002, 2003, 2005) et Petit (2004) ont montré que la période qui a précédé 1971 a connu des propositions de politiques européennes sur le supérieur. La question de l'université européenne a été posée dès le premier jour de la construction européenne. Cette partie retrace les influences de la Commission sur les politiques universitaires entre 1950 et 1998. Elle met en évidence le rôle que les États lui ont accordé et les alliances qu'elle a nouées avec les représentants d'université pour acquérir sa légitimité.

L'histoire des politiques européennes du supérieur a fait l'objet d’interprétations qui se sont cantonnées dans une lecture fonctionnaliste d'abord, selon laquelle le supérieur doit satisfaire les besoins en ressources humaines des Communautés européennes; dans une lecture marquée par l'institutionnalisme historique ensuite ; l'évolution des politiques du supérieur a enfin été étudiée comme un cas de changement de politique promu par des entrepreneurs politiques (Corbett 2003). Cette histoire n'est pas continue. Même s'ils divergent sur les dates où il faut placer les taquets historiques et sur le nombre de périodes qui permettent de rendre les évolutions intelligibles, plusieurs auteurs repèrent des soubresauts, des accélérations brutales et de longues périodes de calme. Les perturbations apparaissent aux moments où la Commission européenne tente d'être associée au pilotage du supérieur, et chacune de ses tentatives suscite des réactions en tous sens. Pour d'aucuns (Davies 2003 ; Brine 1995 ; Field 1998), l'action de la Commission dans le supérieur s'est affirmée au gré de la construction européenne; pour d'autres (Ertl 2006 ; Hingel 2001), elle a été concomitante avec l'adoption ou la révision des Traités; pour d'autres encore (Froment 2003; Corbett 2002), elle a évolué au gré des programmes communautaires sur le supérieur et de l'avancée du projet d'université européenne. Pour étudier l'évolution du rôle de la Commission dans le supérieur, il est aussi possible d'établir une périodisation en partant des enjeux politiques. Jusqu'en 1998, l'histoire de l'implication de la Commission dans le supérieur peut être écrite autour de cinq enjeux, qui ont nourri autant de projets aboutis ou avortés : l'enjeu atomique et de la création de l'université européenne, de l'intégration européenne, de l'harmonisation des études, du pilotage du supérieur et de la compétitivité (Croché 2009). La Commission ne saute pas d'un enjeu à un autre, les chevauchements peuvent être longs et significatifs.

\subsection{Le projet d'université européenne}

L'histoire des politiques universitaires européennes commence le $1^{\text {er }}$ juin 1955 (Corbett 2005). Au cours d'une rencontre des ministres des affaires étrangères des six pays initiateurs des Communautés européennes, le représentant de la République fédérale d'Allemagne a annoncé le souhait de son pays que soit créée une université 
européenne (Martino 1959). L'objectif poursuivi par le gouvernement allemand était de mettre fin à l'isolement scientifique de ses universités, méprisées par une large partie de la société allemande, et d'ouvrir davantage les structures académiques aux talents. Ce projet a suscité des réactions vives de la part des ministres des affaires étrangères de Belgique et des Pays-Bas, qui ont estimé que le sujet n'entrait pas dans l'agenda du jour. D'autres propositions ont suivi, comme celle émise par la France en 1956 que soit créé un Institut pour la recherche et la formation dans les sciences nucléaires (Corbett 2003, 2006), ou celle de créer un centre et une école conjointe de recherche atomique comme bases d'une université européenne. Les discussions qui ont accompagné ces propositions ont débouché sur l'insertion de deux articles dans le Traité Euratom de 1957 : l'article 9 stipule qu' «il sera créé une institution de niveau universitaire dont les modalités de fonctionnement seront fixées par le Conseil statuant [...] sur proposition de la Commission»; l'article 126 que «les propositions de la Commission [...] seront adressées au Conseil dans un délai d'un an à compter de l'entrée en vigueur du Traité ». Ces articles attestent que la réflexion sur le supérieur a commencé au sein des Communautés avant 1971. Dès la fin des années 1950, l'ambition de renforcer l'attractivité des universités des pays des Communautés et la volonté de les inciter à innover étaient évidentes en Europe. Les réactions au projet de créer une université européenne ont toutefois montré que les États restaient attachés à leurs prérogatives. Le Traité de Rome de 1957 n'a fait aucune référence directe à l'enseignement, seules les questions de reconnaissance des diplômes sont abordées dans l'article 57 qui stipule que «le Conseil, sur proposition de la Commission [...], arrête, [...] des directives visant à la reconnaissance mutuelle des diplômes, certificats et autres titres ».

Malgré que le Traité de Rome ait réaffirmé les prérogatives des États en matière d'éducation, en 1958, le Conseil des Communautés a débattu de la manière dont les universités pourraient être mobilisées pour faire avancer l'intégration européenne. Les gouvernements ont aussi envisagé la façon dont l'Europe pourrait les aider à accroitre la qualité de leurs institutions universitaires et de recherche (Corbett 2004a). Les six pays avaient des modèles d'universités très différents et le Traité Euratom les incitait à poser un choix. Ainsi, en mai 1958, les pays ont discuté pour la première fois des articles 9 et 216 du Traité et ont consulté les recteurs européens sur le projet de créer une institution universitaire européenne. Méfiants envers un supranationalisme européen et souhaitant continuer de pouvoir bénéficier des marges de liberté que leurs gouvernements leur laissaient, les recteurs se sont opposés au projet. Le Conseil européen a toutefois créé un groupe de travail chargé de réfléchir au rôle des universités dans la construction européenne qui a conclu ses travaux en déclarant qu'une université européenne était un moteur nécessaire à l'intégration européenne. La proposition émise par le groupe que l'université soit dotée d'un conseil d'administration chargé de faire un rapport régulier à la Commission européenne a suscité l'opposition des pays qui n'ont pas accepté qu'un contrôle puisse être exercé par la Commission sur une université. Peu de temps après, l'idée selon laquelle l'Université européenne avait besoin d'être liée aux 
sciences nucléaires a été abandonnée. Le projet d'université a été détaché de ses objectifs initiaux, la réflexion a depuis ce moment été essentiellement associée à celle de l'intégration européenne. En octobre 1959, l'idée d'une Université européenne centrée sur les sciences humaines a été proposée. Le projet était de créer une Université, appelée à renforcer le potentiel culturel et scientifique de l'Europe et à enseigner les disciplines pertinentes pour l'intégration. Dans le schéma proposé, un Conseil des ministres composé de représentants des six pays devait avoir le contrôle de cette université. La proposition de confier le pilotage de l'université aux pays était en opposition avec celle émise un an plus tôt d'en donner le contrôle à la Commission. Le cycle qui a vu des tentatives pour établir une université supranationale s'est achevé en 1961, quand les chefs d'État et de gouvernement réunis à Bonn ont rappelé que l'éducation était une matière nationale (Corbett 2003). Ils ont décidé que c'était aux ministres de l'enseignement de se réunir et de conclure des conventions intergouvernementales sur le supérieur (Corbett 2006). Les ministres ne se sont toutefois pas souvent réunis au sein des Communautés (Corbett 2005), ils leur ont préféré le Conseil de l'Europe. Dans les années 1950, l'image du Conseil de l'Europe avait été rehaussée lorsque les recteurs, réunis depuis 1959 au sein de la Confédération des recteurs européens (CRE), et les ministres de l'enseignement avaient commencé à travailler avec le Conseil pour la Coopération Culturelle du Conseil de l'Europe. Sans intention intégrationniste, le Conseil de l'Europe ne constituait pas une menace pour les ministres qui auraient pu craindre une perte de souveraineté (Croché 2009), il leur a permis de se réunir pendant quelques années et d'adopter des résolutions sur le supérieur (Haigh 1970). C'est la première organisation à avoir élaboré des textes et des instruments qui permettent les reconnaissance mutuelles des périodes d'études et des diplômes qui ont facilité les mobilités en Europe dès 1953.

L'année 1969 a vu réapparaitre la question universitaire. Le ministre français de l'éducation, Edgar Faure, a lancé un appel aux autres pays pour que le Conseil de l'Europe les aide à résoudre la crise que traversaient les universités, suite aux événements de mai 1968. Il a proposé que soient créés un centre européen d’information et un institut européen de technologie, il a suggéré que les réformes dans le domaine de l'éducation soient coordonnées à l'échelon européen. Une nouvelle fois, l'Europe était appelée à aider les pays à résoudre leurs problèmes nationaux. Cela étant, le Conseil de l'Europe n'a pas suivi Faure. Des ministres de l'enseignement ont alors commencé à émettre des réserves sur le fonctionnement intergouvernementaliste du Conseil de l'Europe (Corbett 2005). Les critiques les plus virulentes ont culminé au sommet de La Haye de 1969, au cours duquel les chefs d'État ont discuté de la relance de l'intégration européenne. À La Haye, les recteurs ont accepté que les Communautés soient dotées d'une capacité de formuler des politiques qui affecteraient les universités (Rüegg 1999), la France a souhaité que se mette en place une coopération européenne en matière d'enseignement en précisant que sa structure d'accueil ne pourrait être le Conseil de l'Europe (Corbett 2003). Au sommet de La Haye, le supérieur était appelé à devenir 
plus important dans la foulée des décisions prises de travailler pour une union économique et monétaire. Le Parlement a profité de l'occasion pour faire adopter une résolution qui a suggéré à la Commission de soumettre une proposition pour la création d'un conseil des ministres de l'enseignement qui travaillerait avec la Commission (Corbett 2005).

À l'issue de l'examen de ce premier enjeu, nous pouvons suivre Corbett (2005) pour qui des « entrepreneurs politiques» (Kingdon 1995) ont voulu offrir à la Commission la possibilité d'agir sur le supérieur. Leurs actions n'ont pas porté leurs fruits, les États se sont opposés au projet de créer une université européenne pilotée par la Commission. $\mathrm{Au}$ terme de cette période, les cartes ont été redistribuées, les États ont voulu que les ministres de l'enseignement se réunissent au sein des Communautés au détriment du Conseil de l'Europe (Croché 2009).

\subsection{L’intégration européenne}

L'organisation d'un Conseil des ministres de l'enseignement à la fin des années soixantes a modifié le paysage institutionnel. Ce ne sont plus les ministres des affaires étrangères qui ont la charge de la coopération en matière d'enseignement mais les ministres compétents qui ont reconnu qu'une coordination de leurs initiatives était souhaitable. En novembre 1971, réunis pour la première fois au sein des Communautés, ils se sont accordés sur le principe d'un programme commun d'action. Un accord intergouvernemental, de bien peu d'ambition quand on le compare au projet initial de création d'une université européenne, a été trouvé sur le projet de créer un institut universitaire européen à Florence. Puisque les ministres de l'enseignement ont convenu que la coopération dans leur domaine de compétence était un corollaire logique de la coopération en matière de formation, dès 1973, certains membres de la CRE (Confédération des recteurs européens) ont constitué un Comité, chargé de la coopération avec la Commission européenne avec laquelle ils n'ont plus cessé de travailler.

Ces diverses actions montrent que les premières initiatives en matière d'enseignement ont concerné le supérieur en vertu d'une conception fonctionnaliste de la société où l'intégration européenne pouvait être accélérée si les élites étaient convaincues qu'elle était désirable (Papatsiba 2001). En 1972, le vice-président de la Commission a indiqué «qu'une politique $[\ldots]$ de l'éducation doit être mise en œuvre [...] dans la Communauté » (Commission européenne 1972, cité in Petit 2002). Le rapport rédigé en 1973 par Janne à la demande de la Commission a donné le ton des réformes à installer dans le domaine de l'enseignement et a accordé la priorité au supérieur. Un Comité de l'éducation a été créé en 1973, qui a regroupé des représentants des États et des hautsfonctionnaires, chargés de préparer les positions des ministres de l'enseignement (Corbett 2004a). La Commission a ensuite eu l'occasion de jouer un rôle plus large depuis sa réorganisation suite à l'élargissement des Communautés au Royaume-Uni, à l'Irlande et au Danemark en 1973. L'enseignement a été mis en lien avec la recherche et la science 
et une nouvelle Direction générale pour la recherche, la science et l'éducation a été créée. La création d'une «Europe de l'enseignement» a commencé en 1974 avec le développement du Comité (Rubio 1997, cité in Ertl 2006). Les actions des années septantes ont été entreprises parce qu'une «compétence implicite » a été reconnue à la Commission (Delgrado \& Losa 1997, cité in Ertl 2006).

À la fin de cette période, la Commission a commencé à intervenir dans le supérieur en étant officiellement chargée de travailler dans ce domaine parce qu'un Conseil et un comité éducation ont été créés au sein des Communautés. Elle a tiré sa puissance de sa collaboration avec la CRE avec laquelle elle a établi des alliances au détriment du Conseil de l'Europe.

\subsection{L'harmonisation des études}

Le travail de préparation dont les étapes sont rappelées ci-dessus s'est concrétisé par l'adoption d'une première résolution sur l'enseignement, le 6 juin 1974. Même si elle affirme que «l'harmonisation des systèmes et des politiques ne peut être considérée comme un but en soi », elle prône l'intensification de la coopération entre les établissements, la reconnaissance des périodes d'études effectuées à l'étranger, la mobilité des étudiants et des professeurs. Le texte ne dit rien de la manière dont ces objectifs doivent être poursuivis, ce qui laisse leur pleine liberté aux autorités nationales. En février 1976, une seconde résolution a inséré ces priorités dans un programme d'action. C'est au cours de cette année qu'un programme pilote d'échanges universitaires a été lancé (Megie \& Ravinet 2004), précurseur d'ERASMUS adopté en 1987. Ces résolutions ont fixé les objectifs que la coopération entre les pays dans le domaine de l'enseignement a poursuivis pendant les décennies suivantes. Les directives de 1974 et 1976 ont conduit à un rapprochement des programmes nationaux qui préparent les étudiants à certaines professions (médecins, dentistes, vétérinaires, pharmaciens, architectes), en listant les connaissances que les diplômés devaient avoir acquises à l'issue de leur formation. Une autre directive a été adoptée en 1988, qui a visé l'installation d'un système de reconnaissance des diplômes du supérieur qui sanctionnent des formations professionnelles d'une durée minimale de trois ans (European Council 1988) et qui voulait permettre à tout citoyen d'exercer sa profession dans n'importe quel pays de la Communauté.

À la fin de cette troisième période, la Commission a pris des résolutions pour faciliter les reconnaissances, conformément au rôle qui lui avait été donné par le Traité de Rome.

\subsection{Le pilotage de l'enseignement supérieur}

Jusqu'en 1985, aucune disposition légale n'autorisait la Commission à intervenir dans les politiques d'enseignement. L'arrêt Gravier de 1985, qui a inclus le supérieur dans le 
champ de la formation professionnelle où elle est habilitée à intervenir, a fourni une base juridique aux initiatives de la Commission et lui a permis d'intervenir dans l'enseignement (Milner 1998). En 1986, l'Acte unique a fait entrer le mot « université » dans le Traité de Rome qu'il vient renforcer « par l'inclusion de dispositions relatives à l'importance de la cohésion économique et sociale» (European Commission 1994). À partir de ce moment, les autorités européennes ont dégagé des moyens pour former les ressources humaines nécessaires à la réalisation du grand marché. Les années 1987 à 1990 ont été marquées par le lancement des programmes communautaires comme ERASMUS, destiné à soutenir la mobilité étudiante via le système de crédits ECTS (European Credit Transfer System) qui a ensuite constitué un des axes du processus de Bologne. Il est indubitable qu'il a permis à la Commission de devenir l'organisation la plus active en Europe dans la stimulation de la mobilité (Smith 1996). Avec ses compétences limitées dans l'enseignement, la Commission n'était pas en position d'imposer une réforme des systèmes (Van der Wende 2001). Elle a agi à la marge, et le programme ERASMUS est considéré comme celui qui a le mieux fonctionné de ceux qui ont été initiés par l'Union dans le domaine de l'enseignement (Teichler 2001). Le succès de ce programme (Altbach \& Teichler 2001) a fait oublier les réticences des ministres à son égard et a reçu le soutien des associations d'universités. Les académiques ont joué un rôle important dans le développement de la mobilité (Smith 1980). Les programmes de mobilité ont rendu l'idée d'intervention de la Communauté sur les systèmes éducatifs non pas acceptable mais populaire (Papatsiba 2005).

À la fin de la décennie 1980, le projet européen s'est précisé. Une résolution a été adoptée en 1988 par les ministres de l'enseignement qui y prônaient le développement d'une dimension européenne dans l'éducation pour renforcer l'identité européenne (Papatsiba 2005). Le Mémorandum sur l'enseignement supérieur dans la Communauté européenne (1991) a défini le rôle de la Commission comme celui d'un « agent catalyseur facilitant les actions communes et la coopération dans le respect du principe de subsidiarité » (European Commission 1991). La question de l'enseignement a ensuite conservé une place importante dans le débat public sur la construction de l'Europe avec la signature du Traité de Maastricht (1992) qui a créé l’Union européenne et établi la libre circulation des biens, des services et des personnes. Si ce Traité a réaffirmé la souveraineté nationale sur les systèmes éducatifs, l'article 126, en vertu duquel «la Communauté contribue au développement d'une éducation de qualité », a autorisé l'Europe à y intervenir de façon supplétive. La réaffirmation du respect des prérogatives nationales dans les textes fondateurs serait la contrepartie de la mise en œuvre d'autres outils visant en réalité à les contourner (Cussó 2004). Ce Traité a consolidé la base légale sur laquelle la Commission peut s'appuyer (De Wit \& Verhoeven 2001). Depuis Maastricht, les politiques économiques et éducatives ont été de plus en plus associées dans les rhétoriques européennes. En 1993, la Commission a émis l'idée d'un espace européen scientifique et éducatif. Trois projets qui préparent Bologne ont été adoptés sous forme de programmes en 1995 : Socrates, Leonardo Da Vinci, Jeunessse pour l'Europe. 
De 1985 à 1995, la question du pilotage du supérieur s'est posée avec acuité. Soutenue par la Cour de justice des Communautés européennes qui a rendu des avis en sa faveur, par la CRE avec laquelle elle avait fait alliance, la Commission a pu adopter des programmes qui ont élargi ses activités. Après avoir concerné les reconnaissances, ses politiques ont visé la mobilité et les liens entre l'enseignement et le monde du travail.

\subsection{L'enjeu de la compétitivité et l'annonce du processus de Bologne}

Le Traité qui a été adopté à Amsterdam en 1997 a accordé une place importante à l'enseignement. Peu de temps après, le 11 novembre 1997, dans sa communication Pour une Europe de la connaissance, la Commission (1997) a réaffirmé sa volonté de créer un espace européen qui ne serait pas centré seulement sur les qualifications. Constatant que «nous entrons désormais dans la société de la connaissance », la Commission a estimé qu'il fallait développer une véritable «Europe de la connaissance » qui soit compétitive. Elle envisageait que les systèmes d'enseignement nationaux évoluent en douceur vers l'intégration et qu'elle soit appelée à jouer un rôle central dans le pilotage de l'ensemble communautaire ainsi constitué. La partie qui suit montre que les termes qui ont été utilisés par la Commission européenne en 1997 ressemblent à ceux de la déclaration de la Sorbonne de 1998. Les États d'Europe se sont réapproprié le projet de la Commission européenne pour le supérieur.

\section{Le processus de Bologne : analyse de l'intervention croissante de la Commission européenne dans le supérieur entre 1998 et 2009}

Avant 1998, les liens entre les systèmes d'enseignement d'Europe étaient établis notamment grâce aux programmes communautaires (Haug \& Race 1998). À la fin de la décennie 1990, les projets de l'Union dans le supérieur étaient toujours dominés par les restrictions imposées par le principe de subsidiarité et le refus qu'opposaient les États à l'harmonisation. À l'intérieur de la Commission, des tensions existaient aussi, qui portaient sur la question de savoir quelle part le supérieur devait jouer dans le passage de la communauté économique européenne à une Union politique (Neave 2003). Cette partie veut mettre en évidence les méthodes douces par lesquelles la Commission européenne est parvenue à prendre progressivement le pilotage des politiques universitaires en Europe alors qu'elle a été explicitement exclue du processus lancé à la Sorbonne en 1998 par des États qui voulaient conserver leurs prérogatives dans le domaine éducatif. 


\subsection{De la Sorbonne à Prague : les luttes pour le pilotage de la réforme des universités}

Réunis le 25 mai 1998 à la Sorbonne à l'initiative du ministre français de l'éducation nationale, Claude Allègre, les ministres de l'enseignement supérieur d'Allemagne, d'Italie, de France et du Royaume-Uni ont signé une déclaration par laquelle ils ont annoncé leur projet « d'harmoniser l'architecture du système européen d'enseignement supérieur». L’objectif affiché qu'ils poursuivaient était de rendre leurs systèmes d'enseignement supérieur plus attrayants sur la scène internationale (Charlier 2009; Charlier \& al. 2009). En même temps qu'ils signaient la déclaration de la Sorbonne, les ministres prenaient aussi des mesures nationales et attendaient de l'harmonisation qu'elle les aide à résoudre leurs problèmes. La déclaration de la Sorbonne n'est donc pas, pour ceux qui l'ont signée, l'expression d'un réel projet politique européen (Charlier \& Croché 2007).

Les organisations non gouvernementales et internationales n'ont pas été associées à la rencontre de la Sorbonne. La Commission européenne a été explicitement exclue du projet initié par quatre pays qui souhaitaient conserver leur pouvoir sur leur enseignement. En 1997, la Commission a voulu créer un « espace éducatif européen ouvert et dynamique », en 1998, Allègre visait à installer un " espace européen ouvert de l'enseignement supérieur ». La déclaration de la Sorbonne mobilise le même lexique que la Commission pour proposer un projet plus ambitieux. Cette proximité nous conduit à formuler l'hypothèse selon laquelle Allègre s'est réapproprié le projet de la Commission (Croché 2009). Allègre considérait que la collaboration dans le supérieur ne pouvait passer que par des accords entre des gouvernements assumant leur souveraineté sur leur système éducatif. Il n'a pas accepté les modalités par lesquelles la Commission entendait construire un espace européen du supérieur. Il ne pouvait imaginer qu'elle en soit l'initiatrice. La coopération intergouvernementale garantissait des marges maximales de liberté aux pays, un processus communautaire aurait tendu à les réduire. Allègre a ainsi fait rupture par rapport à une évolution longue. Avant lui, les États ont plusieurs fois fait appel à des structures européennes, Commission ou Conseil de l'Europe, quand leur enseignement se trouvait face à un problème qu'ils n'arrivaient pas à résoudre avec leurs ressources politiques nationales. Ils étaient prêts à troquer une part de leur souveraineté contre l'aide que leur apporterait une instance internationale pour résoudre leurs problèmes nationaux. Allègre a changé l'équation. Comme ses prédécesseurs, il a sollicité le palier international pour l'aider à faire passer les réformes qui lui semblaient essentielles. Il n'était pas prêt pour autant à négocier quoi que ce soit ou à offrir quelque chose en échange du service qu'il sollicitait. Il a voulu installer un mécanisme de coopération intergouvernementale, qui laisserait les mains libres aux États : en fonction de leurs besoins de l'heure, ils auraient ou non activé un palier international de concertation qui aurait été sans autonomie ni capacité d'initiative. Fondamentalement, 
ce n'aurait été rien d'autre qu'un contre-feu destiné à arrêter l'avancée de la Commission.

La déclaration de la Sorbonne se terminait par un appel aux autres pays d'Europe à rejoindre le processus. Cette invitation a suscité des réactions vives de la part de ministres qui n'ont pas accepté que quatre pays imposent un modèle d'enseignement pour l'Europe. Les ministres de l'éducation des pays de l'Union se sont donc réunis en conseil informel en octobre 1998 à Baden, en Allemagne, pour un échange libre sur le fond de la déclaration de la Sorbonne et sur la manière dont la réunion dont elle était le fruit avait été préparée et organisée. Une controverse est ainsi apparue sur le projet de créer un espace européen du supérieur, la question qui était posée était de savoir s’il fallait faire passer le supérieur dans les matières européennes ou le laisser dans les matières nationales. Les ministres présents à Baden ont estimé qu'une étude comparative devait être entreprise sur les structures d'enseignement supérieur en Europe (Hackl 2001). Cette demande, qui constituait un désaveu très clair de l'initiative des signataires de la Sorbonne, a été rapidement soutenue et prise en charge par la Commission européenne. Voyant par là une manière d'intégrer le processus, elle a commandé une étude de faisabilité du projet de la Sorbonne à la CRE et à la CRUE (Confédération des conférences de recteurs de l'Union européenne). Ce faisant, elle a aidé les ministres divisés sur l'appel de la Sorbonne à accepter de coopérer. Le Conseil informel de Baden a aussi décidé de constituer un groupe de suivi informel de la rencontre de la Sorbonne, composé des directeurs généraux de l'enseignement supérieur des pays appelés à assurer les prochaines présidences de l'Union, à savoir l'Autriche, l'Allemagne, la Finlande, du pays qui s'est engagé à organiser le prochain sommet, i.e. l'Italie, des pays signataires de la déclaration de la Sorbonne non repris dans la liste ci-dessus, i.e. le Royaume-Uni et la France. La CRE et la CRUE, chargés de l'étude de faisabilité (rapport Trends I), et la Commission y ont également été conviés (Hackl 2001). La constitution de ce groupe est un moment essentiel du processus puisque la Commission s'est imposée dans son suivi.

Incluse dans le groupe de suivi, la Commission n'avait toutefois pas encore la capacité d'imposer ses règles du jeu. Le bras de fer qu'elle a engagé avec Allègre dès 1998 a été particulièrement visible lors du sommet de Bologne des 18 et 19 juin 1999 au cours duquel les ministres du supérieur de 29 pays d'Europe ont signé une déclaration par laquelle ils ont annoncé leur volonté de créer, par la coopération intergouvernementale, un espace européen de l'enseignement supérieur pour 2010. Si elle a largement contribué à la réussite du projet initié à la Sorbonne, la Commission a de nouveau été exclue du processus dit de Bologne par 29 ministres qui ont réaffirmé leur compétence sur leur enseignement. La déclaration de Bologne a été nettoyée du terme harmonisation, présent dans la déclaration de la Sorbonne, qui aurait pu suggérer une intégration significative des systèmes d'enseignement. Elle a aussi été expurgée des références à l'Union européenne et à la Commission, qui a tenté de faire inscrire ses projets dans le texte de la déclaration (Zgaga 2004, cité in Racké 2006). Depuis Bologne, le processus regroupe des pays qui ne font pas partie de l'Union, ce qui limitait les chances de la Commission 
d'en prendre le pilotage. À l'issue du sommet, Allègre a choisi la ville de Prague pour accueillir le sommet suivant, ce qui a profondément déplu à la Commission qui l'a fait savoir d'une manière vive.

Malgré l'épisode de révolte frondeuse contre la Commission lors du sommet de Bologne, l'adhésion massive qu'a suscitée la déclaration et l'importance des travaux à entreprendre pour 2010 ont peu à peu conduit les États à discuter de la place qu'ils allaient accorder à la Commission dans le processus. Les critiques acerbes des États opposés à l'intervention de la Commission se sont alors adoucies, le groupe de travail qui s'était créé après la Sorbonne s'est réuni en juillet 1999 et a souligné que le rôle de la Commission était primordial dans le processus (Hackl 2001). Forte du soutien de plusieurs pays, la Commission a alors commencé à influer plus lourdement sur les décisions. En juillet 1999, ses projets de réformes pour le supérieur et les moyens (notamment financiers) qu'elle peut mettre à la disposition des pays pour faire évoluer leurs projets ont été reconnus comme bénéfiques pour l'avancée du processus. Depuis ce moment et même si le processus de Bologne ne concerne pas que l'Union européenne, le poids qu'elle a représenté dans le processus lui a donné une capacité d'influence déterminante, renforcée encore par le fait que les ministres de l'enseignement se rencontrent de manière régulière lors de Conseils formels ou informels (Croché 2009). Leurs réunions contribuent à les doter de conceptions identiques des problèmes que rencontrent les institutions et des manières opportunes de les prendre en charge. Elles produisent ou renforcent les référents doctrinaux et idéologiques qui guident l'orientation des décisions. C'est lors de ces réunions que la Commission a pu agir pour influencer les États-membres de l'Union qui représentaient la moitié des pays inclus dans le processus de Bologne. Après la réunion de Bologne, les ministres de l'éducation des pays de l'Union se sont réunis de façon informelle à Tampere en septembre 1999, de façon formelle en novembre 1999 à Bruxelles.

La période qui a suivi le sommet de Bologne a été riche en structuration du processus. Si les universités ont été intégrées au développement du processus grâce à la Commission qui leur a commandé une étude de faisabilité en 1998, il en a été de même des associations d'étudiants qui ont été consultées par le Conseil des ministres de l'éducation des pays de l'Union dès 2000 dans le même temps qu'ils ont été chargés par la Commission de diffuser l'information sur le programme ERASMUS auprès des étudiants. Le sommet qui a réuni trente-deux pays d'Europe à Prague le 19 mai 2001 pour faire le bilan du travail accompli et pour définir les orientations prioritaires du processus pour les années à venir a poursuivi le travail de structuration. Les ministres ont installé une structure de suivi composée d'un groupe de suivi et d'un groupe préparatoire. « Le groupe de suivi est composé de représentants de l'ensemble des signataires, des nouveaux participants et de la Commission européenne, et est présidé par l'État membre qui exerce la présidence de l'Union européenne. Le groupe préparatoire comprend les pays qui ont organisé les précédentes conférences ministérielles et [le] pays chargé d'organiser la prochaine [...], deux États-membres et deux États non membres de 
l'Union européenne; ces quatre derniers représentants sont élus par le groupe de suivi. L'État qui exerce la présidence de l'Union européenne et la Commission européenne font également partie du groupe préparatoire. Le groupe préparatoire est présidé par le représentant du pays qui accueillera la prochaine conférence ministérielle. L'Association de l'université européenne (EUA) [issue de la fusion de la CRE et de la CRUE en 2001], l'Association européenne des établissements d'enseignement supérieur (EURASHE), l'ESIB [...] et le Conseil de l'Europe seront consultés dans le cadre des travaux de suivi» (Communiqué de Prague 2001). Le sommet de Prague est donc celui de l'intégration des partenaires non gouvernementaux. "Les ministres se sont engagés à poursuivre leur coopération [...] en profitant de toutes les possibilités offertes par la coopération intergouvernementale, [...] ainsi que par les programmes communautaires » (Ibid.). Cette phrase montre le pas qui a été franchi depuis 1999. À Bologne, les ministres avaient retiré toute référence au travail de l'Union, deux années plus tard, il est fait une mention explicite des programmes communautaires dans le communiqué adopté par les ministres. Des débats se sont aussi déroulés à Prague sur les limites de l'espace européen de l'enseignement supérieur : « les ministres ont souhaité la bienvenue aux pays auxquels les programmes communautaires Socrates et Leonardo Da Vinci ou Tempus-Card sont ouverts et qui ont demandé à rejoindre le processus de Bologne » (Ibid.). À ce moment, cette formulation confuse a l'ambition de suggérer que les frontières de l'espace européen de l'enseignement supérieur sont celles que délimitent ces trois programmes communautaires installés par la Commission.

Avec le recul, le sommet de Prague apparaît comme un compromis, un moment ambigu de structuration. Le projet politique des initiateurs de la déclaration de la Sorbonne y a trouvé un prolongement concret lorsque des représentants des autorités académiques et des étudiants ont été intégrés aux groupes de pilotage, il a été trahi quand le Conseil de l'Europe et la Commission y ont été accueillis (Croché 2006). L'ouverture consentie aux organisations non gouvernementales et internationales reste mesurée : elles obtiennent une voix consultative et ne sont pas considérées comme des partenaires définitifs, alors que les pays sont membres à part entière du processus et ont une voix délibérative. La Commission a une position intermédiaire : elle ne dispose pas d'une voix délibérative mais est considérée comme un membre à part entière et donc définitif. Parce qu'elle s'est, au contraire d'Allègre, intéressée à tous les acteurs qui participent au processus et parce qu'elle s'est efforcée d'offrir à chacun les garanties et les avantages qui avaient le plus de valeur pour lui, la Commission est parvenue à se faire accepter comme une organisation centrale du processus, capable d'intervenir sur ses orientations (Croché 2009). Prague est ainsi le moment d'apogée du processus. Il s'est ensuite fait absorber par la stratégie de Lisbonne, lancée en mars 2000 pour faire de l'Europe l'économie de la connaissance la plus compétitive au monde, qui lui a fixé de nouveaux repères dont il avait besoin pour prolonger les dynamiques qu'il avait initiées. 


\subsection{De Prague à Louvain : contrôle de la réforme des universités par la Commission}

Après Prague, le processus de Bologne a fait des émules. En octobre 2001, les directeurs généraux de la formation professionnelle des pays de l'Union réunis à Bruges ont affirmé leur volonté d'élever la qualité de la formation professionnelle pour améliorer la compétitivité de l'économie européenne et renforcer la reconnaissance mutuelle des qualifications entre les pays. Ce projet a été légitimé par le Conseil de mars 2002 au cours duquel les chefs d'État réunis à Barcelone ont souhaité que « des actions semblables au processus de Bologne, mais adaptées au domaine de l'enseignement et de la formation professionnels » soient lancées. Répondant à cet appel, réunis à Copenhague le 30 novembre 2002, les ministres de l'éducation et de la formation professionnelle de 31 pays d'Europe et la Commission ont adopté une déclaration visant à « accroître la coopération dans le domaine de l'enseignement et de la formation professionnelle» (Copenhagen Declaration 2002). À partir de ce moment, les processus de Copenhague et de Bologne ont été considérés comme des outils importants pour la réalisation des objectifs qui ont été assignés à l'éducation et à la formation par la stratégie de Lisbonne. La Commission que le Traité de Rome a chargée de poursuivre la coopération entre les États dans le domaine de la formation professionnelle a vu le processus de Copenhague comme une aubaine. Elle a pu montrer aux acteurs inclus dans le processus de Bologne qu'elle pouvait agir dans un processus connexe et qu'il serait judicieux pour les pays que des liens soient établis entre les processus. Les acteurs de Bologne se trouvent confrontés au processus de Copenhague, piloté par la Commission, dont ils sont forcés de tenir compte puisque, depuis Prague, le processus de Bologne a reconnu sa compétence sur l'éducation tout au long de la vie.

Le communiqué qui a été adopté par 40 pays à Berlin en septembre 2003 a fixé de nouvelles limites à l'espace européen de l'enseignement supérieur. Seuls peuvent en faire partie les pays qui ont signé la " convention culturelle européenne », adoptée sous l'égide du Conseil de l'Europe en 1954. Les conditions que les pays doivent respecter pour pouvoir s'inscrire dans le processus délimitent un espace qui s'apparente désormais plus au Conseil de l'Europe qu'à l'Union européenne. Concurrencée sur les questions des frontières de l'espace européen du supérieur, la Commission a pourtant réussi à faire inscrire un grand nombre de ses projets dans le processus. Lors du sommet de Berlin, les ministres ont confirmé la constitution des groupes de pilotage et le rôle particulier de la Commission dans le processus. Ils ont adressé un appel à peine voilé à cette dernière lorsqu'ils ont annoncé que pour atteindre leurs objectifs, un important soutien, y compris financier, et des décisions appropriées de la part des gouvernements nationaux et des organismes européens, seraient nécessaires. À Berlin, l’impact de la stratégie de Lisbonne sur le processus de Bologne est devenu aussi visible que considérable : « les Ministres tiennent pleinement compte des conclusions du Conseil européen de Lisbonne (en 2000) et de Barcelone (en 2002) [...] qui nécessitent de développer davantage d'activités et une coopération plus étroite dans le contexte du processus de Bologne» (Communiqué de Berlin 2003). Depuis Berlin, les ministres du supérieur ont 
considéré que le projet lancé en 2000 par l'Union européenne de construire un espace européen de la recherche, est appelé à constituer le second pilier de l'économie de la connaissance, et que des liens serrés et nombreux doivent être établis avec l'espace européen de l'enseignement supérieur. La contribution significative que la recherche effectuée dans les établissements d'enseignement supérieur apporte et peut apporter au progrès économique, est considérée comme indéniable. La décision d'intégrer le doctorat au processus, tel que proposé par la Commission et l'EUA, a toutefois contribué à remettre sur le travail les sujets qui fâchent. La structuration des études en trois cycles « 3-5-8 « n’apparaissait pas dans les premiers textes (Sorbonne, Bologne, Prague) qui ont mis en avant les deux cycles de base et la formation tout au long de la vie sans évoquer le doctorat. Le calibrage du doctorat en trois ans modifie le statut et la signification de l'épreuve. La tradition universitaire européenne valorisait le doctorat long, fruit d'une ascèse solitaire au terme de laquelle le doctorant était coopté par ses pairs. Le doctorat en trois ans est fatalement inscrit dans le projet global d'un laboratoire et n'a plus rien d'une épreuve solitaire. Cette nouvelle manière de faire ne vise pas à alimenter les universités, qui produisent suffisamment de docteurs pour assurer le renouvellement de leur personnel académique et scientifique, elle veut contribuer à essaimer des compétences de recherche dans les milieux professionnels pour en faire évoluer la culture dans un sens favorable à l'innovation et à la recherche, comme le suggère la stratégie de Lisbonne.

Depuis 2003, la Commission (2003) entend piloter la réforme des universités via son soutien aux politiques de recherche. Sa communication sur «Le rôle des universités dans l'Europe de la connaissance » est le premier texte qu'elle a publié dans lequel elle affiche les orientations des réformes des universités qu'elle appelle de ses vœux. La relance de la stratégie de Lisbonne en mars 2005 lui a donné une raison supplémentaire de s'occuper des universités. Depuis 2005, la production de communications de la Commission sur les universités et sur leur contribution à la stratégie de Lisbonne s'est accélérée, dans l'intention de susciter une grande mobilisation. Le titre de la communication qu'elle a publié en avril 2005, « Mobiliser les cerveaux européens : permettre aux universités de contribuer pleinement à la stratégie de Lisbonne » (Commission européenne 2005a) ne laisse aucun doute sur le rôle qu'elle attribue aux universités dans le renforcement de la compétitivité de l'Europe. Elle estime que les universités sont essentielles pour atteindre les objectifs de Lisbonne, mais qu'elles ne sont pas en mesure de mettre tout leur potentiel au service de ce projet. «L'Europe doit renforcer les trois pôles du triangle de la connaissance, à savoir l'éducation, la recherche et l'innovation. Les universités sont essentielles dans chacun des trois domaines. Investir davantage et mieux dans la modernisation et la qualité des universités revient à investir directement dans l'avenir de l'Europe et des européens » (Ibid.).

Le sommet qui s'est tenu à Bergen en mai 2005 et qui a rassemblé les ministres de 45 pays d'Europe et d'Asie a donné à la Commission européenne la légitimité pour guider la réforme des universités. Les ministres ont élargi les groupes de pilotage du processus en accueillant les syndicats des enseignants rassemblés sous l'égide de l'IE (Internationale de l'éducation), l'UNICE (Union des confédérations industrielles et des 
employeurs de l'Europe) et l'ENQA (Association européenne pour l'assurance de la qualité dans l'enseignement supérieur). L'inclusion de ces trois organisations n'a pas été de nature à bouleverser les équilibres puisqu'elles étaient déjà acquises à la cause de la Commission européenne (Croché 2009). La rencontre de Bergen a donc prolongé les projets de la Commission et a partiellement concrétisé ses ambitions de contrôler le supérieur. Au cours le la rencontre, les ministres ont adopté un cadre global de qualifications pour l'enseignement supérieur qui décrit les différents cycles et les différents domaines d'études en termes de compétences, intégrant par là le projet de la Commission. Ils se sont aussi fixé de nouvelles priorités en recommandant notamment que les programmes doctoraux promeuvent la formation interdisciplinaire et le développement de compétences transférables. À Bergen, les ministres ont octroyé le droit de vote à la Commission dans les structures de pilotage du processus. Elle bénéficie désormais du même statut que les pays, alors que les autres acteurs du processus ont conservé un statut consultatif, elle peut désormais voter dans un processus d'européanisation lancé dans un domaine qui reste de compétence nationale. Cette décision n'a suscité aucune opposition alors que l'accès au droit de vote était symboliquement et techniquement très important pour la Commission. Ce moment peut donc être considéré comme l'apothéose pour la Commission qui a lutté pendant des décennies pour qu'il en soit ainsi. Elle a offert à ses partenaires du processus les moyens financiers dont ils avaient besoin pour poursuivre leur collaboration (Croché 2006) et a procuré à chacun ce qu'il souhaitait: les organisations non gouvernementales (ESIB, EUA, EURASHE, IE, UNICE) sont considérées comme des partenaires de la prise de décision sur le supérieur, le Conseil de l'Europe a vu se concrétiser ses projets d'avancer plus loin dans la reconnaissance des diplômes, les pays ont obtenu les outils qui leur ont permis de faire évoluer leurs projets et sont beaucoup plus attractifs qu'ils ne l'étaient avant 1998 (Charlier \& Croché 2007).

La période qui a suivi le sommet de Bergen a été riche en initiatives de la Commission, soutenue par les États-membres de l'Union, pour moderniser les universités et les faire contribuer à l'innovation. Lors du sommet d'Hampton Court du 27 octobre 2005, les chefs d'État ont demandé à la Commission de déterminer, dans le secteur des universités, les domaines d'action pouvant faire progresser l'agenda sur la croissance et l'emploi pour que l'Europe puisse en tirer des avantages. Il est entendu que les travaux n'aboutiront pas à de nouveaux processus, mais s'intégreront dans les dispositifs existants, ce qui laisse supposer que les actions de la Commission sur les universités passeront par le processus de Bologne.

Dans la foulée du sommet d'Hampton Court, le Conseil éducation s'est réuni le 15 novembre 2005 pour un échange de vues sur les actions à entreprendre en faveur des universités de haut niveau. Le 12 décembre 2005, suite à la demande des chefs d'État, la Commission (2005b) a remis un rapport dans lequel elle souligne qu'il faut s'intéresser à la gestion des universités, à la manière dont elles transfèrent au marché l'innovation et les compétences, qu'il faut améliorer et diversifier le financement des 
universités et assurer une coopération plus étroite entre le monde universitaire et celui des entreprises. Confortant le rapport de la Commission, le Conseil de l'Union européenne (2006a) réuni en mars 2006 a demandé le renforcement des actions au niveau européen pour la mise en œuvre des réformes nécessaires dans les universités et la recherche d'ici à la fin de 2007. Il a invité les États à faciliter l'accès des universités à des sources de financement complémentaires, y compris privées, et à lever les obstacles à la constitution de partenariats public-privé avec les entreprises. En mai 2006, la Commission $(2006 \mathrm{a}, 2006 \mathrm{~b})$ a publié deux communications. Elle s'y dit prête à soutenir la modernisation des universités, grâce à un processus d'inventaire et de partage de bonnes pratiques, et à ses programmes de financement de l'éducation, de la recherche et de l'innovation. Elle souligne qu'il faut démanteler les barrières autour des universités en Europe: "les efforts réalisés dans le contexte du processus de Bologne visent la convergence des structures et de la durée des programmes d'études. [....] Ceci ne crée pas en soi les conditions pour une mobilité intra-universitaire accrue. Un effort de grande envergure devrait être consenti pour que les réformes fondamentales du processus de Bologne soient en place dans tous les pays de l'UE en 2010 ». Les communications de la Commission ont été des sources d'inspiration pour le Conseil de l'Union (2006b) de juin 2006 au cours duquel les chefs d'État ont demandé qu'il soit donné suite aux communications de la Commission sur les défis que devront affronter les universités. Ils encouragent les États à promouvoir l'excellence, à favoriser la modernisation, la restructuration et l'innovation dans le supérieur.

Ces longues descriptions montrent que la Commission entend imposer une réforme majeure des universités et en commander la direction et le rythme, sans que cela ne suscite l'opposition des États. Ces derniers adhèrent à la lecture de la Commission, ils se disent favorables à une réforme de leurs universités et semblent prêts à en confier le pilotage à la Commission. Il n'est donc plus seulement question de lui donner la conduite du processus de Bologne, il est aussi envisagé de faire appel à son expertise pour réformer les universités.

Le débat sur la modernisation des universités a croisé celui initié en 2005 sur la création de l'Institut européen de technologie (IET). À la faveur de la relance du processus de Lisbonne, en février 2006, la Commission (2006c) a présenté une communication dans laquelle elle envisageait la mise en place d'un IET chargé de renforcer les liens entre les trois pôles du «triangle de la connaissance». L'objectif affiché était de rendre l'Union compétitive en comblant le fossé qui sépare le monde académique du monde industriel. Cette communication et celles qui ont suivi ont été mal accueillies par la communauté scientifique et universitaire (Daumas 2006) qui s'est fortement opposée aux propositions de la Commission de détacher ses meilleures équipes de recherche au profit de l'IET pendant 10 ans et de faire de l'IET un acteur européen de la recherche qui délivrerait ses propres diplômes. Après d'âpres débats entre les universités et la Commission, un règlement portant création de l'IET a été adopté en mars 2008 par le Parlement européen et le Conseil. Il ne délivrera pas de diplômes, ceux-ci seront décer- 
nés par les établissements d'enseignement supérieur qui participent à l'IET. Cette disposition indique la victoire des opposants au projet de voir une institution d'enseignement et de recherche supranationale décerner des diplômes. Les mêmes causes produisent les mêmes effets. Si la Commission est parvenue à guider la réforme des universités, certains États et universités sont restés farouchement opposés, comme ils l'avaient été en 1957 avec le projet d'université européenne, à la création d'une institution supranationale capable de délivrer des diplômes reconnus par les pays.

L'épisode de révolte lié à la création de l'IET n'a pas réduit la puissance de la Commission. Le sommet de Londres qui a rassemblé 46 pays en avril 2007 a été marqué par des discussions sur la création après 2010 d'un secrétariat permanent du processus piloté par la Commission européenne ou le Conseil de l'Europe. La question du passage du supérieur dans les compétences européennes a donc cessé d'être un tabou, comme l'atteste aussi le débat sur le processus de Bologne qui s'est tenu le 6 mars 2008 au Parlement européen et au cours duquel certains parlementaires ont plaidé pour que le supérieur devienne une compétence communautaire. Cette position qui faisait partie des discours «politiquement incorrects » il y a dix ans a cessé d'être marginale, des recteurs et des représentants étudiants ont déclaré qu'ils la partageaient. Peu après, les documents publiés au sommet qui s'est tenu à Louvain-la-Neuve et à Leuven en avril 2009 montrent que les acteurs du processus de Bologne se sont réappropriés les prescriptions de la Commission, notamment son idée de «triangle de la connaissance ». Une modification apparaît toutefois, les références à la Commission sont présentes mais ses prescriptions sont réappropriées par les acteurs qui y font référence sans plus mentionner systématiquement leur concepteur. Les prescriptions de la Commission ont ainsi des effets structurants, elles agissent désormais d'elles-mêmes.

\section{Conclusion : l'université au service du triangle européen "Éducation, recherche, innovation "}

Les universités qui se sont créées en Europe il y a quelques siècles n'existent plus. De nouvelles missions et fonctions leur ont été attribuées, après qu'elles aient été soumises à des incitations amenées par des projets divers, dont le processus de Bologne et la stratégie de Lisbonne sont les plus importants. Jusqu'en 2003, la Magna Charta Universitatum (CRE 1988), un texte adopté en 1988 par les universités européennes qui voulaient célébrer leurs valeurs traditionnelles, est resté le seul texte de référence qui s'imposait aux universités en matière de politique universitaire. Dès 2003, la Commission a alors jugé bon de produire des communications sur les universités, sans consulter l'EUA, tentant par là d'imposer les orientations des réformes qu'elle souhaitait voir entreprendre.

Depuis le sommet de Prague (2001), le processus de Bologne est un espace de négociation orienté de façon significative par la Commission européenne. Son influence sur les politiques d'enseignement supérieur se lit dans les idées véhiculées par les acteurs in- 
clus dans les instances de décision du processus de Bologne ou dans les lieux de débat qui lui sont proches et dans les intérêts aujourd'hui défendus par les divers protagonistes. Plus la Commission a été intégrée au processus, plus elle a pu peser sur son pilotage, plus la vision de l'université et de l'Europe qui s'est propagée rejoint les propositions qu'elle a formulées dans ses communications sur le rôle des universités. Les déclarations de la Sorbonne (1998) et de Bologne (1999) prônaient une université humaniste dans une « Europe du savoir»; cette conception s'est rapidement estompée, elle n'est plus défendue depuis 2003 et on n'en trouve plus aucune trace dans le projet de 2005 de créer un Institut Européen de Technologie (IET), qui, souvenons-nous, avait déjà été proposé par Faure en 1969 (cf. I.1). L’Université est désormais envisagée comme une institution qu'il est stratégique de mobiliser pour relancer la croissance économique européenne et l'emploi, elle doit s’intégrer au triangle de la connaissance « Éducation, Recherche, Innovation » censé contribuer à la compétitivité de l'Europe sur la scène mondiale et libérer le potentiel d'innovation européen. Sa mission principale est de produire des élites (interdisciplinaires), une préoccupation qui a pendant longtemps été très mal portée, au moins en Europe continentale. Un basculement entre la préoccupation honteuse pour les élites en Europe et l'affirmation triomphante de la nécessité de réformer tout le système pour améliorer leur formation a toutefois eu lieu (Croché 2009). Les élites sont l'objet de toutes les attentions, des programmes communautaires ont été lancés à tous les niveaux du parcours scolaire pour pouvoir les sélectionner dès les années nonantes. Le programme communautaire ERASMUS Mundus lancé en 2003 ou les bourses starting grant de l'European Research Council en donnent de parfaites illustrations: dans les deux cas, il est question d'offrir aux meilleures intelligences des conditions matérielles séduisantes pour qu'elles se localisent en Europe. La formation des élites est donc désormais pensée en réseau, des réseaux internationaux sont suscités pour former des individus hautement qualifiés.

Depuis 2005, la Commission européenne entend définir le «bon enseignement», la «bonne université », mais aussi la « bonne science » en réseau. Je dois à Jean-Émile Charlier d'avoir attiré mon attention sur l'idée selon laquelle la recherche scientifique peut être considérée comme le cheval de Troie que la Commission a fait pénétrer dans les universités sans guère rencontrer de résistance. Elle attribue des budgets considérables à un nombre limité d'équipes de recherche, ce qui attise les concurrences et oriente la recherche vers les questions qu'elle estime prioritaires et vers les manières de travailler qu'elle promeut. Les universités européennes ont globalement accepté le jeu proposé par la Commission, les centres de recherche tentent à la fois de devenir de plus en plus concurrentiels et de multiplier les partenariats qui les valorisent. Ils s'alignent sur les prescriptions de la Commission européenne telles qu'elles apparaissent dans l'European Research Council créé en 2007. Depuis 2005, une nouvelle conception de la science est mise en avant par les instances européennes. La science est désormais définie en réseau, les seules équipes qui peuvent concourir pour bénéficier des financements octroyés par la Commission sont celles qui sont déjà connectées à d'autres, en Europe et dans le 
reste du monde. La valeur d'une équipe se définit en partie par la qualité et la variété de ses partenaires. La disjonction entre recherche et enseignement en devient très logique : autant des établissements relativement autarciques ou associés à la grandeur d'un peuple, d'une nation, étaient tenus de tenir le rang le plus élevé tant en recherche qu'en enseignement, autant cette nécessité s'efface dans le fonctionnement en réseau. Dans celui-ci, il s'agit de s'allier avec les partenaires qui sont les plus et les mieux complémentaires. Un établissement peut donc se révéler particulièrement efficace en se spécialisant dans quelques aspects d'une des fonctions attribuées aux universités et en s'adossant à ses partenaires pour briller dans toutes les autres : l'enseignement des résultats des travaux d'un laboratoire de pointe ne doit par exemple pas nécessairement en être assuré par les responsables, il peut être plus efficace qu'il le soit par des collègues qui se sont spécialisés dans la transmission de connaissances de haut niveau. Une division du travail intellectuel et scientifique est ainsi encouragée dans l'objectif de maximiser la productivité de chacun des éléments du réseau et du réseau lui-même et de favoriser une programmation ex ante des résultats des programmes de recherche.

\section{Bibliographie sélective}

(L'auteur tient à disposition une bibliographie complète)

Altbach, P.G. et Teichler, U. 2001. Internationalization and exchanges in a globalized university, Journal of studies in international education, Vol.5, $\mathrm{n}^{\circ} 1,5-25$.

Brine, J. 1995. Educational and vocational policy and construction of the European Union, International Studies in the Sociology of Education, Vol.5, $\mathrm{n}^{\circ} 2,145-163$, Routledge, London.

Charlier, J-É. 2009. Le processus de Bologne, son histoire officielle, quelques éléments de son bistoire cachée et quelques enjeux immédiats. In Charlier, J-É.; Croché, S. et Ndoye, A.K. (Eds.) Les universités africaines francophones face au LMD. Les effets du processus de Bologne sur l'enseignement supérieur au-delà des frontières de l'Europe, Academia Bruylant, Louvain-la-Neuve, 23-68.

Corbett, A. 2005. Universities and the Europe of knowledge: ideas, institutions and policy entrepreneurship in European Union higher education policy, 1955-2005, Palgrave, Basingstoke.

Croché, S. 2009. Bologne confisqué. Constitution, autour de la Commission européenne, d'un acteurréseau et d'un dispositif européen de l'enseignement supérieur, Thèse de doctorat, FUCaM \& Université Lumière-Lyon II, Mons/Lyon, 409 pp.

Cussó, R. 2004. Nouvean paradigme éducatif, nouvelle comparaison statistique internationale: Commission européenne et Eurostat, Séminaire Les comparaisons internationales des politiques éducatives, RAPPE, Paris, 24-25 mai. 
Daumas, C. 2006. La Commission précise son projet d'institut européen de technologie. Note 2006/64, 3 mars

Davies, P. 2003. Widening Participation and the European Union: direct action-indirect policy. European Journal of Education, Vol.38, n¹, 99-116, Wiley.

De Wit, K. et Verhoeven, J. 2001. The higher education policy of the European Union: with or against the member states. In Huisman, J.; Maassen, P.A.M. et Neave, G. (Eds.) Higher Education and the Nation State, Elsevier-Pergamon, Oxford, 175-233.

Ertl, H. 2006. European Union policies in education and training : the Lisbon agenca as a turning point?, Comparative Education, Vol.42, n 1, 5-27, Chicago.

Field, J. 1998. European Dimensions. Education, Training and the European Union. Jessica Kinglsley Publishers, London.

Froment, E. 2003. The European Higher Education Area: A New Framework for the Development of Higher Education, Higher Education in Europe, Vol.XXVIII, N¹, 2731, Taylor \& Francis Journal, London.

Hackl, E. 2001. Towards a European Area of Higher Education: change and convergences in European Higher Education, European University Institute, San Domenico.

Haigh, A. 1970. À Ministry of Education for Europe. London: Geo Harrap.

Haug, G. et Race, J. 1998. Inter-regional Cooperation in Higher Education in Europe, Journal of Studies in International Education, Vol.2, $\mathrm{n}^{\circ} 2,3-34$.

Hingel, A.J. 2001. Education Policies and European Governance - Contribution to the interservice groups on European governance, European Journal for Education Law and Policy, Vol.5, n¹-2, 7-16, Springer, Netherlands.

Kingdon, J.W. 1995. Agendas, Alternatives and Public Policy, Second edition, Little Brown \& Co, Boston.

Megie, A. et Ravinet, P. 2004. Contrainte de coopération intergouvernementale et processus d'européanisation: la construction des espaces européens de l'enseignement supérieur et de la justice, Papier présenté à la journée d'études sur l'Européanisation des politiques publiques et intégration européenne, Paris, février.

Meyer, J.W.; Ramirez, F.O.; Rubinson, R. et Boli-Bennett, J. 1977. The World Educational Revolution 1950-1970, Sociology of Education, Vol.50, 242-258, American Sociological Association.

Milner, S. 1998. Training policy. Steering between divergent national logics. In Hine, D. et Kassim, H. (Eds.) Beyond the market: the EU and national social policy. Routledge, London, 156-177. 
Neave, G. 2003. The Bologna declaration: some historic dillemas posed by the reconstruction of the community in Europe's systems of Higher Education, Educational Policy, Vol.17, n¹, 141164.

Papatsiba, V. 2005. Higher Education and student mobility in the EU: action and rationale, Paper presented at the third conference on knowledge and politics, University of Bergen, 18-20 May.

Petit, I. 2004. Politique européenne d'éducation et rhétorique économique: un reflet des contraintes juridico-politiques du cadre actionnel de la Commission européenne. Note de recherche $\mathrm{n}^{\circ} 2$, Institut d'études européennes de l'Université de Montréal et de l'Université McGill, Montréal.

Racké, C. 2006. The Bologna process and the EU: neither within nor without. Contribution à la troisième conférence internationale Euredocs. Centre for Higher Education Research, University of Kassel, 16-18 juin. Consulté le 22/03/2008 à l'adresse http://euredocs.sciences-po.fr/en/conference/2006/euredocs06Rack\%E9.pdf

Rüegg, W. 1999. La CRE : autonomie et cadre européen, CRE-Action, n¹15

Smith, A. 1980. From'Euphoria' to Pragmatism: towards a new start for higher education cooperation?, European Journal of Education, Vol.1, n¹, 77-95.

Smith, A. 1996. Regional cooperation and mobility in a global setting: the example of the European Community. In Blumenthal, P.; Goodwin, C.; Smith, A. et Teichler, U. (Eds.) Academic mobility in a changing world, Jessica Kingsley, London.

Teichler, U. 2001. Changes of ERASMUS under the umbrella of SOCRATES, Journal of Studies in International Education, Vol.39, n 4, 5-26.

Van der Wende, M. 2001. The international dimension in national higher education policies: what has changed in Europe in the last five years?, European Journal of Education, Vol.36, $\mathrm{n}^{\circ} 4$,

431-441. 